\title{
Correction to: Primary Pediatric Brain Tumors of the Posterior Fossa Part II: A Comprehensive Overview of Medulloblastoma
}

\author{
Lisa Liang, Christopher Aiken, Kathleen Felton, Amanda Hogg, \\ Frank van Landeghem, T. Klonisch, David D. Eisenstat, \\ and Tamra E. Werbowetski-Ogilvie
}

\section{Correction to:}

Chapter 16 in: H. Marzban (ed.), Development of the Cerebellum from Molecular Aspects to Diseases, Contemporary Clinical Neuroscience, https://doi.org/10.1007/978-3-319-59749-2_16

The original version of this article was inadvertently published with some errors in figure-2. The same has been updated later as below.

1. In the Group 3 yellow column, MYCN has been updated as "MYC."

2. In the Group 3 yellow column, "de differentiation" has been updated as "de-differentiated."

3. In the Group 4 green column, "isochormosome" is incorrectly spelled. This has been corrected as "isochromosome".

4. Followed consistency in the word "signaling" throughout figure.

5. In the Group 4 green column, the ratio 3:1 has been updated as 2:1.

6. Page 339, second paragraph, 3rd last line has been corrected to refer "Figure 2."

The updated online version of this chapter can be found at https://doi.org/10.1007/978-3-319-59749-2_16 